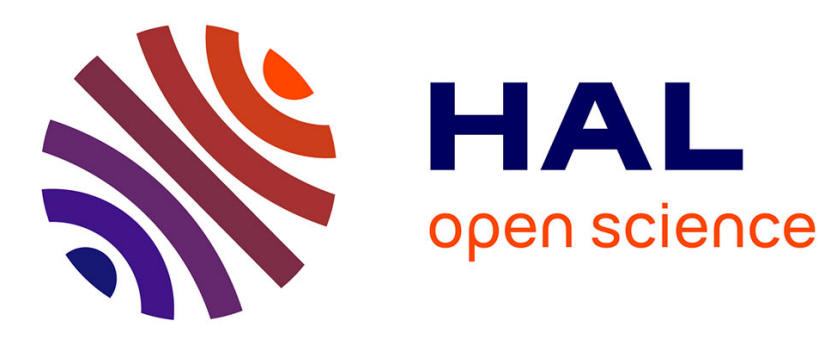

\title{
A new inspection robot for pipelines with bends and junctions
}

\author{
Swaminath Venkateswaran, Damien Chablat
}

\section{To cite this version:}

Swaminath Venkateswaran, Damien Chablat. A new inspection robot for pipelines with bends and junctions. Tadeusz Uhl. Advances in Mechanism and Machine Science, 73, Springer, Cham, pp.33-42, 2019, Mechanisms and Machine Science, 978-3-030-20130-2. 10.1007/978-3-030-20131-9_4 . hal02098350

\section{HAL Id: hal-02098350 \\ https://hal.science/hal-02098350}

Submitted on 4 Jun 2019

HAL is a multi-disciplinary open access archive for the deposit and dissemination of scientific research documents, whether they are published or not. The documents may come from teaching and research institutions in France or abroad, or from public or private research centers.
L'archive ouverte pluridisciplinaire HAL, est destinée au dépôt et à la diffusion de documents scientifiques de niveau recherche, publiés ou non, émanant des établissements d'enseignement et de recherche français ou étrangers, des laboratoires publics ou privés. 


\title{
A new inspection robot for pipelines with bends and junctions
}

\author{
Swaminath Venkateswaran ${ }^{1}$ and Damien Chablat $^{2}$ \\ 1 Centrale Nantes, Laboratoire des Sciences du Numérique de Nantes (LS2N), \\ UMR CNRS 6004, 1 rue de la Noë, 44321 Nantes, France \\ swaminath. venkateswaran@ls2n.fr, \\ 2 CNRS, Laboratoire des Sciences du Numérique de Nantes (LS2N), \\ UMR CNRS 6004, 1 rue de la Noë, 44321 Nantes, France
}

\begin{abstract}
The application of robots for the inspection of pipelines are of greater significance in industries such as nuclear, chemical and sewage. The major problem in the design of these robots lies in the selection of a suitable locomotion principle, selection of an articulation unit that facilitates the robot to pass through pipe bends and management of cables. In this context, the design of a new bio-inspired piping inspection robot that resembles an elephant trunk has been presented. With the help of leg mechanisms and actuators, a caterpillar locomotion is used within this trunk for establishing adaptive contact points with the walls of pipeline. For the passage through bends and junctions, several case studies of existing researches have been taken into account for the design of an articulation unit. Two solutions, (i) a passive tensegrity structure and (ii) an active tensegrity structure have been proposed for the robot to pass through pipe bends and junctions. A detailed design analysis of the passive solution that uses a universal joint has been presented in this article.
\end{abstract}

Keywords: Piping inspection robot, Bio-inspired, Elephant trunk, Tensegrity structure, Universal joint

\section{Introduction}

Manual inspection and maintenance of pipelines in industries such as nuclear and chemical are not only time consuming but also pose threat to human life and causes long term radiation effects. Piping inspection robots play an important role in such situations and they have a major attraction in the scientific research community. The main design criteria for these robots are locomotion, contact type with walls, cable management and articulation unit to pass through bends. Roh et al. [1] proposed a classification of in-pipe inspection robots viz. Pig type, Wheel type, Caterpillar type, Wall-press type, Walking type, Inchworm type,and Screw type. This classification provides a glimpse of the contact type with walls and the locomotion principle of the robot. However, detailed research on the design of an articulation system for bends and cable management inside 
complicated pipeline trajectories have not been carried out. In this article, we focus mainly on the key issues to be addressed for a piping inspection robot to pass through bends and junctions. Based on that, two articulation units have been proposed. These systems can be classified as active and passive units. For example, Hirose et al. [2] proposed THES-I robot which employs a universal joint actuated by a DC-Motor. Anthierens et al. [3] developed an electro pneumatic robot which employs a metallic bellow system to pass through bends. A steering mechanism powered by servo-motors was designed by Ankit et al. [4] to work through pipe bends. However, some interesting passive systems include the robot developed by Kwon et al. [5] where a compression spring is employed to push and pull the two motor modules when working through T-sections and bends. Zhang et al. [6] also proposed a robot with a passive flexible helical axle articulation unit. A bio-inspired piping inspection robot has been presented in [7] and [8]. By coupling leg mechanisms with linear actuators, the robot moves like a caterpillar and establishes a tight contact with the walls of pipeline [7] . However, the robot is incapable of working inside junctions and bends as it is a rigid prototype. In this article, the design of this caterpillar robot has been completely modified into a reconfigurable system. The new design of the robot resembles the trunk of an elephant having articulation units as well as the caterpillar modules being retained for establishing contact points at all instances of locomotion. Two tensegrity structures have been proposed for the articulation unit. For the first one, a passive universal joint with tension loaded springs at $120^{\circ}$ has been designed for the robot to pass through bends. The spring system tends to limit the bending angle of the universal joint to a certain value. The second one incorporates cables to drive the mechanism to facilitate the robot to travel inside pipe bends and junctions. This article explains in detail the design and analysis of the first solution and brief discussions are made for the second solution.

\section{Design factors and constraints for developing a new architecture}

Recently, Henry et al. and Chablat et al. [7-9] have proposed a bio-inspired piping inspection robot that is capable of inspecting pipelines between diameter range of 40-94 $\mathrm{mm}$. The schema of the robot proposed in [7] is represented in Fig. 1. By using slot-follower leg mechanisms and linear actuators, the robot accomplishes its locomotion as well as contact with pipeline walls in 6 steps. However, the major disadvantages of this robot are it is a rigid prototype and the management of cables have not been studied in detail.

\subsection{Factors to be considered for linear, bent and branched pipes}

In general, for the design of a piping inspection robot, three main considerations have to be taken into account which are rectilinear motion, passive compliance, and active compliance. The architecture represented in Fig. 1 addresses the first 


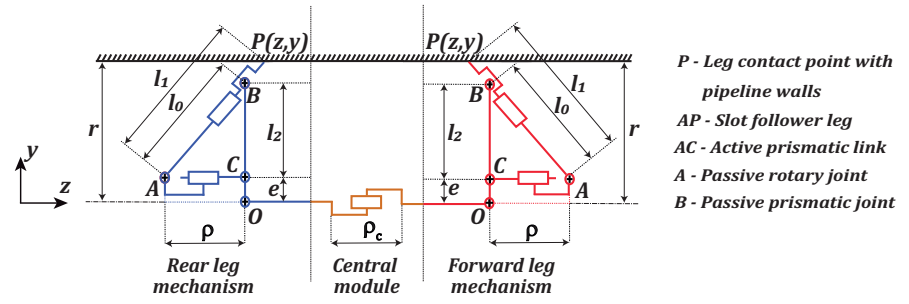

Fig. 1. Schema of the existing bio-inspired piping inspection robot ${ }^{\dagger}$

criteria as it holds good inside perfectly vertical and horizontal pipelines. It is essential to address the other two criteria in order to make the robot work through bends and junctions. Passive compliance could be imagined by a robot that passes through bends at $90^{\circ}$ or less. The articulation unit that drives the robot through such bends need not be necessarily active. Examples of such robots include the squirm pipe robot proposed by Zhang et al. [6] and the caterpillar type robot proposed by Kwon et al. [5]. For active compliance, it is essential for the articulation unit as well as the contact mechanism with walls to be actuated and adaptable. Robots passing through T-branches and junctions need to adopt active compliance. However, the direction of movement inside a T-branch or junction is usually controlled through software programming and sensors. An interesting example is the robot proposed by Ryew et al. [10] which employs a double active universal joint (actuated by two motors and two clutches) where the articulation remains active while passing through junctions and remains passive through an elbow. This robot also uses a wheel system with a pantograph mechanism to have contact with pipeline walls which adapts according to bends and straight profiles. The general representation of the passive and active compliance is shown in Figs. 2a and 2b. Another important factor for the articulation unit is the maximum angle at which it bends when encountering a curvilinear profile. Depending on the center line radius (CLR) of the pipe bend, the articulation unit can facilitate the robot to bend either at $x^{\circ}$ or $-x^{\circ}$ which could be referred to as "Tilt". The tilting range of $\pm x^{\circ}$ is depicted in Fig. 2c.

\subsection{Architecture of the new piping inspection robot}

In order to address the compliance factors and tilt limits, a new architecture of the bio-inspired robot has been proposed and it is represented in Fig. 3. The caterpillar locomotion from [7] has been retained in this architecture. This module ensures tight contact with pipeline walls through a force control algorithm inside vertical and horizontal orientations. By the introduction of suitable articulation units between each motor modules, the robot can be made to pass through pipe bends. While moving through bends, the entire robot resembles an

\footnotetext{
$\dagger 3 \mathrm{D}$ model of this robot in CATIA with joints/links and robot simulation available in - https://www.youtube.com/watch?v=7z6-by83mtw
} 

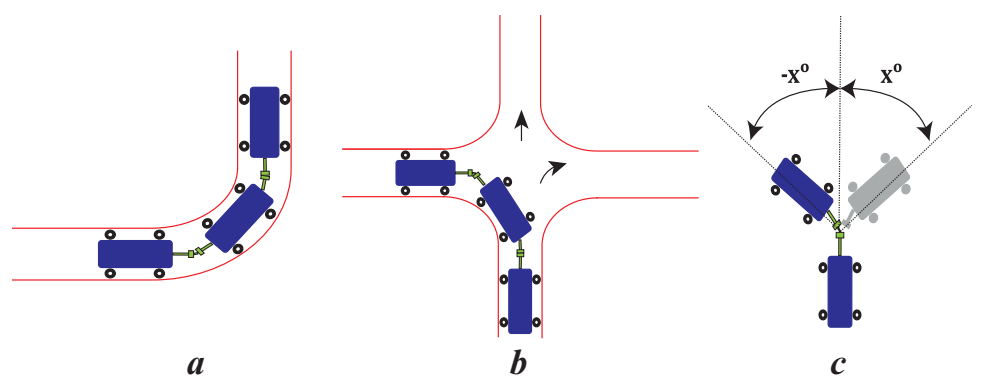

Fig. 2. Typical representation of passive (a) \& active (b) compliances and tilt range (c) for a piping inspection robot

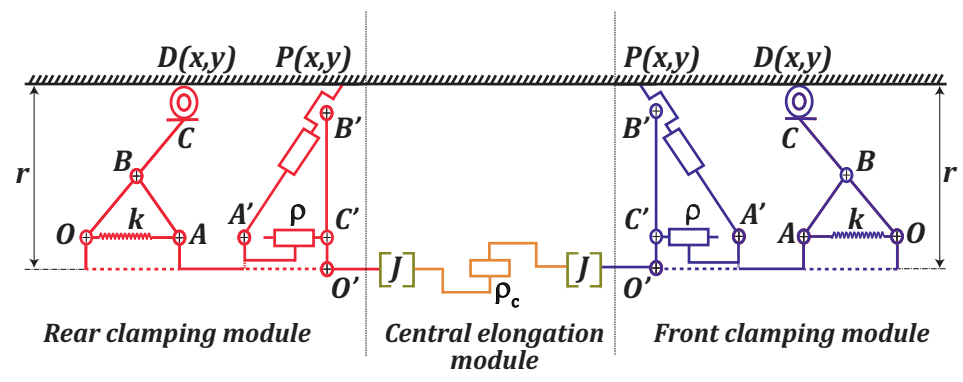

Fig. 3. Schema of the new bio-inspired robot inside a pipeline of radius $r$

"Elephant trunk" wherein the force is transmitted from the front to rear module to pull the robot in order to pass through the curvature and vice-versa. The articulation units are designed with a view that they undergo deflection like a beam without torsion. In Fig. 3, $O^{\prime} A^{\prime} B^{\prime} P$ represents the slot-follower mechanism which is being unchanged. They are attached with the front and rear clamping modules for accomplishing the contact points with pipeline walls. The parameters $\rho$ and $\rho_{c}$ represents the actuation units of the leg and central elongation modules. The joints $J$ represents the articulations that are being introduced in the robot. A spring-loaded four-bar mechanism with a wheel is introduced in the front and rear end of the robot. $O B, B C$ and $A B$ represents the links of four-bar and $O A$ denotes the tension spring having a stiffness of $k \mathrm{~N} / \mathrm{mm}$. This mechanism ensures contact with the walls of the pipeline during static and dynamic modes. The wheel mechanism also helps the robot to easily enter and exit through pipe bends and junctions.

\section{Tensegrity structures as joints for the robot to pass through pipe bends and junctions}

We focus mainly on the introduction of a suitable articulation unit that can facilitate the robot to work through bends at $90^{\circ}$ or less. As the contact point 
criterion for the robot has been addressed by retaining the caterpillar module and introduction of wheel mechanism, we look for an articulation unit to work through bends. Two tensegrity structures have been proposed for articulation unit in this article: a passive structure in order to pass through bends and an active structure to pass through junctions. We go in detail with the former mechanism and its design. A universal joint is proposed for the passive solution. To keep in balance the individual parts of the universal joint assembly, three tension loaded springs at an angle of $120^{\circ}$ are mounted at a radius $r_{f}$ from the central axis of the universal joint. The universal joint transmits the necessary force from the front to the rear end of the robot while passing through bends thereby making the robot to resemble like an "Elephant trunk". The proposed articulation unit is represented in Fig. 4.

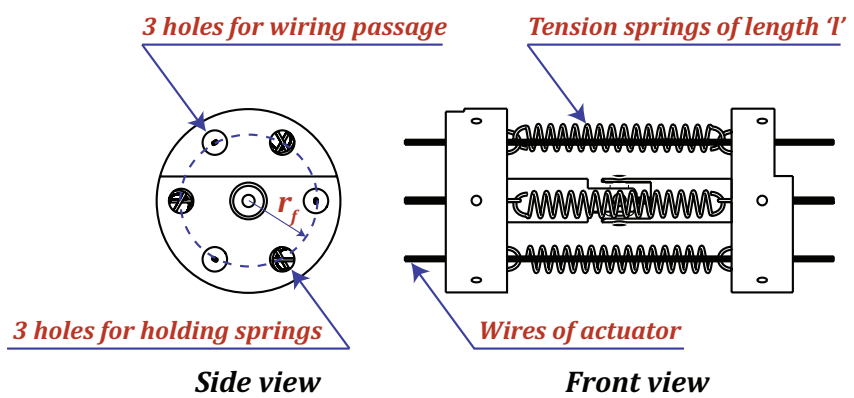

Fig. 4. Proposed tensigrity structure with three springs and a universal joint

In Fig. $4, r_{f}$ represents the radius on the flange where the tension springs are mounted. $l$ represents the free length of each spring. The other three holes of the flange are used as a passage for the wiring coming from the actuators. Fig. 4 also represents the default posture of the joint under non-working condtions as well as inside a perfectly vertical and horizontal pipeline. It is necessary to derive the equations for the joint in order to determine the stable configurations. With the help of SIROPA package in Maple [11,12], the passive universal joint system with the spring is constructed in the form of a parallel manipulator similar to a 3-SPS -U [13] configuration and it is represented in Fig. 5.

In Fig. 5, $\mathbf{B}_{1}, \mathbf{B}_{2}$ and $\mathbf{B}_{3}$ represents the base mounting point for the three springs. $\mathbf{C}_{1}, \mathbf{C}_{2}$ and $\mathbf{C}_{3}$ represents the other end of the mounting point for the springs. A represents the central origin (universal joint). The springs are represented by the three red actuators. The vector coordinates for the base points $B_{i}$ from the center of mounting flange is given by:

$$
\mathbf{B}_{1,2,3}=\left[\begin{array}{c}
r_{f} \cos \left(\frac{2 \pi j}{3}\right) \\
r_{f} \sin \left(\frac{2 \pi j}{3}\right) \\
-r_{f}
\end{array}\right] \quad \text { where } j=0,1,2
$$




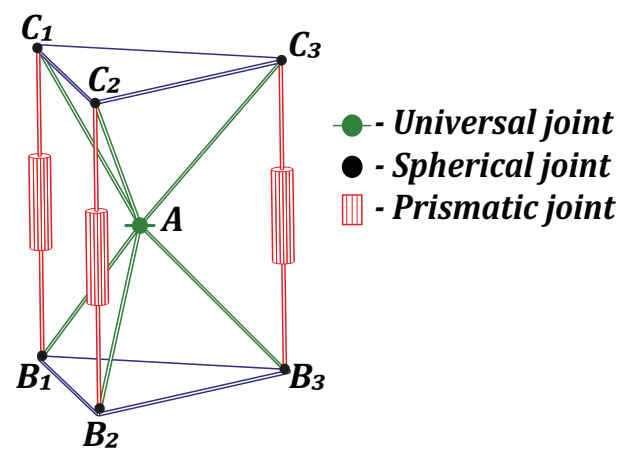

Fig. 5. Correlation of the proposed articulation unit to a parallel manipulator of type 3-SPS -U

In Eq. (1), the minus sign indicates that the base mounting points are on the negative side with respect to origin $\mathbf{A}=[0,0,0]$. For the estimation of mounting points $C_{i}$, the theory of Tilt-torsion (T\&T) is employed. Parallel Kinematic Machines (PKM) adopt the concept of T\&T where the platform of the robot is usually mobile and asymmetric [14]. However, torsion is not considered for the proposed actuation unit. Thus, only the tilt angle and azimuth is essential for determining the position of mounting points $C_{i}$. The representation of tilt and azimuth with the articulation unit is shown in Fig. 6.

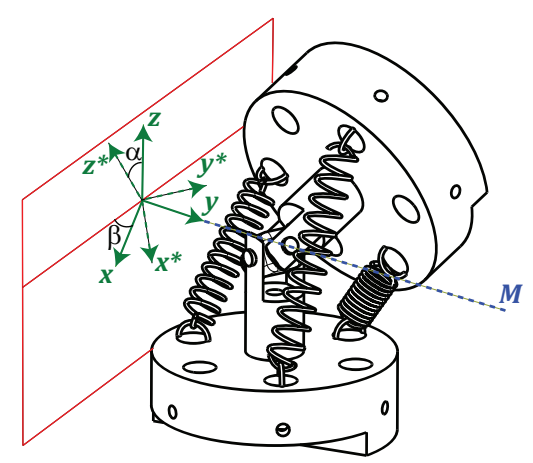

Fig. 6. Representation of tilt $(\alpha)$ and azimuth $(\beta)$ angles on the universal joint

In Fig. 6, $\beta$ represents the azimuth angle between $\mathrm{x}$-axis and the face of $\mathrm{z}$ axis. A rotation of $\alpha$ about axis $M$ causes a shift of $x y z$ axes to $x^{*} y^{*} z^{*}$ axes and this refers to the tilt. The rotation matrix $\mathbf{R}$ for the tilt and azimuth is given by:

$$
\mathbf{R}=\mathbf{R}_{z}(\beta) \mathbf{R}_{x}(\alpha) \mathbf{R}_{z}(-\beta)
$$


When $\alpha$ and $\beta=0$ or in other words when the base and the mobile platform are parallel, Eq. (2) becomes an identity matrix. The vector coordinates for $C_{i}$ in this condition is given by:

$$
\mathbf{C}_{1,2,3}=\left[\begin{array}{c}
r_{f} \cos \left(\frac{2 \pi j}{3}\right) \\
r_{f} \sin \left(\frac{2 \pi j}{3}\right) \\
r_{f}
\end{array}\right] \quad \text { where } j=0,1,2
$$

For finding the exact position of $C_{i}$ at a certain tilt, the rotation matrix obtained from Eq. (2) is multiplied by Eq. (3). The length of the springs $l_{i}$ can now be estimated by calculating the distance between $B_{i}$ and $C_{i}$ and the equation is given by:

$$
l_{i}=\sqrt{\left(B_{i}[1]-C_{i}[1]\right)^{2}+\left(B_{i}[2]-C_{i}[2]\right)^{2}+\left(B_{i}[3]-C_{i}[3]\right)^{2}} \text { with } i=1,2,3
$$

While passing through bends and junctions, it is not necessary for the coupling to bend up to a certain angle. The tension loaded spring mechanisms restricts the bending angle till a certain limit. In order to estimate the limits, it is essential to calculate the feasible workspace for the articulation unit. The tilt angle $\alpha$ is critical when the robot encounters a bend inside pipeline. For ease of calculations, we set the limits for azimuth angle as $\beta \in[-\pi, \pi]$. With the help of Maple, the feasible maximum and minimum workspace range constraint equations $\mathcal{C}$ are estimated which are given by:

$$
\begin{array}{r}
\mathcal{C}_{1+3 i}=\left[2 r_{f}{ }^{2}(-1+\cos (\alpha))(\cos (\beta))^{2}-4 r_{f}^{2} \sin (\beta) \sin (\alpha)\right. \\
\left.+4 r_{f}{ }^{2}-l_{x}{ }^{2}\right] \\
\mathcal{C}_{2+3 i}=\left[\begin{array}{r}
-2 r_{f}{ }^{2} \cos (\beta)(\sin (\beta) \cos (\alpha)-\sin (\beta)-2 \sin (\alpha)) \sqrt{3} \\
-2 r_{f}{ }^{2}(-1+\cos (\alpha))(\cos (\beta))^{2}+4 r_{f}{ }^{2} \sin (\beta) \sin (\alpha) \\
\left.+3 r_{f}{ }^{2} \cos (\alpha)+5 r_{f}{ }^{2}-2 l_{x}{ }^{2}\right] \\
\mathcal{C}_{3+3 i}=\left[2 r_{f}{ }^{2} \cos (\beta)(\sin (\beta) \cos (\alpha)-\sin (\beta)-2 \sin (\alpha)) \sqrt{3}\right. \\
-2 r_{f}{ }^{2}(-1+\cos (\alpha))(\cos (\beta))^{2}+4 r_{f}{ }^{2} \sin (\beta) \sin (\alpha) \\
\left.+3 r_{f}^{2} \cos (\alpha)+5 r_{f}{ }^{2}-2 l_{x}{ }^{2}\right]
\end{array}\right.
\end{array}
$$

where for $i=0,1$ we have $l_{x}=l_{\min }, l_{\max }$

The limits $l_{x}=l_{\min }$ and $l_{\max }$ represents the minimum and maximum extension of each spring while passing through a pipe bend. The radius $r_{f}$ for the mounting points of the spring from flange center is taken as $11 \mathrm{~mm}$ from the existing design [7]. By setting the limits $l_{\min }=7 \mathrm{~mm}$ and $l_{\max }=31 \mathrm{~mm}$, we compute the feasible range of $\alpha$ in Maple and the result is represented in Fig. 7.

In Fig. 7, the blue regions highlight the feasible solutions. The ideal values of tilt $(\alpha)$ lies between $[-\pi / 3, \pi / 3]$. Various postures can be generated for a fixed 


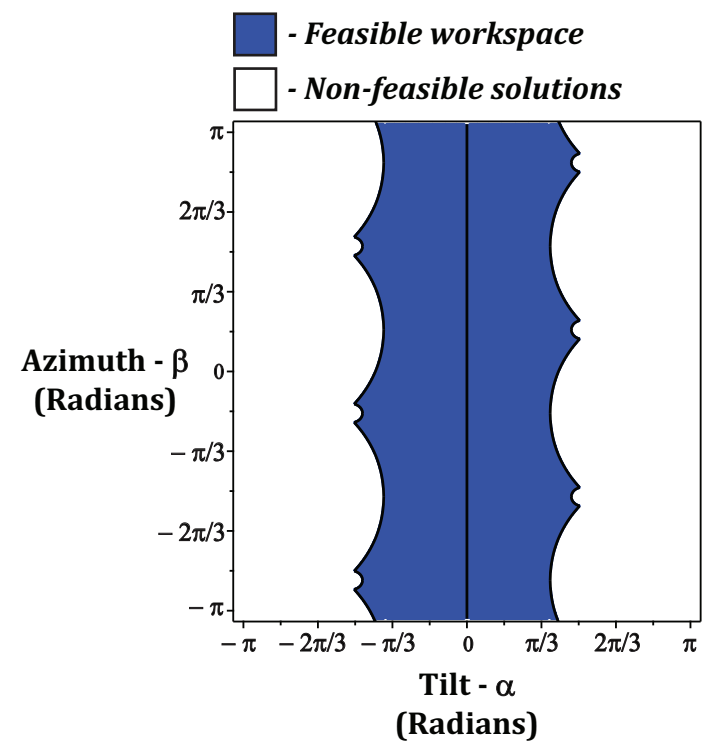

Fig. 7. Plot of workspace for the universal joint with respect to $\operatorname{Tilt}(\alpha)$ and $\operatorname{Azimuth}(\beta)$ angles

value of tilt in this range with azimuth ranging between $[-\pi, \pi]$. However, there also exists very few feasible solutions of tilt between $[-\pi,-\pi / 3]$ and $[\pi / 3, \pi]$ (not shown in Fig. 7) but the azimuth angle is highly limited and there exists collision between parts in this zone. Using the ideal range of tilt and varying azimuth angles, the spring lengths and their limits could be designed and realized in CATIA. The cables coming from the actuators can be made to pass through the holes provided in the flange in a uniform pattern to the central control unit. By assembling the tensegrity mechanism, caterpillar module, four-bar wheel mechanisms, and standard fasteners, the entire robot can be realized in CATIA. The rendered model of the robot which resembles an "Elephant trunk" is represented in Fig. 8.

For the second tensegrity structure, cable mechanisms could be added to the passive solution. The analysis of an actuated X-shape Snelson mechanism was done by van Riesen et al [15]. This mechanism uses a four-bar mechanism with crossed links and tension springs. Cables passing through the springs are used to actuate this mechanism. However, this analysis was done for a 2D structure. By introducing the cables in the passive configuration through the springs, the issue of active compliance could be addressed and when passing through a junction, these cables can be actuated thereby allowing the robot to choose the best path. The static and dynamic force analysis performed by van Riesen et al [15] will be extended to the proposed 3D architecture to determine stable configurations and feasible workspaces. 


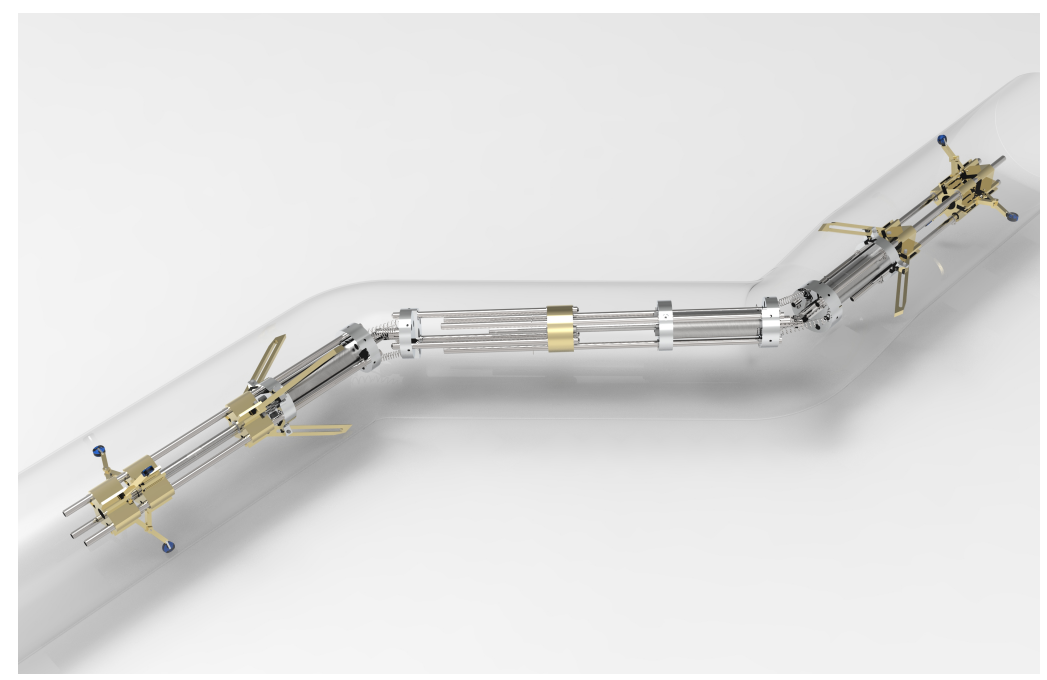

Fig. 8. Rendered model of the new "Elephant trunk" type bio-inspired robot

\section{Conclusions and Discussions}

The key criteria for a piping inspection robot such as passing through curvilinear trajectories and tilt angles have been addressed in detail. The compliances discussed in this article facilitated in the modification of existing caterpillar robot of rigid type into a reconfigurable system that resembles an "Elephant trunk". For the passive configuration, a tensegrity mechanism with universal joint and three tension springs was proposed and for the active configuration, cables are added through the springs to control the same mechanism [16]. The design and analysis of the first solution were studied in detail. The feasible workspace limits were calculated for the universal joint with respect to the tilt and azimuth angles. The results of this analysis helped in realizing a digital prototype of the entire robot in CATIA. The cable management is also addressed by letting the wires of actuators to pass through the holes of flange units. However, it is necessary to make an initial prototype of this robot to understand the configurations and look for alternate solutions if necessary.

In the future, workspace analysis of the universal joint with respect to azimuth angle and spring extension length will be carried out. This calculation will be essential to determine the optimal length and width of actuator units that will be used in the robot when they pass through pipe bends and elbows. A detailed analysis will be done on the second tensegrity structure for addressing the issue of active compliance. For the active tensegrity structures, based on the weight factors and cables, an analysis will be done to determine if a single or stacked model is required for the robot. The design and analysis of four-bar wheel mechanism will also be studied in detail through optimization techniques for determining the dimensions of bars, tension spring, and wheel. 


\section{References}

1. Roh, S. G., \& Choi, H. R. (2005). Differential-drive in-pipe robot for moving inside urban gas pipelines. IEEE transactions on robotics, 21(1), 1-17.

2. Hirose, S., Ohno, H., Mitsui, T., \& Suyama, K. (1999). Design of in-pipe inspection vehicles for $\phi 25, \phi 50, \phi 150$ pipes. In Robotics and Automation, 1999. Proceedings. 1999 IEEE International Conference on (Vol. 3, pp. 2309-2314). IEEE.

3. Anthierens, C., Ciftci, A., \& Betemps, M. (1999). Design of an electro pneumatic micro robot for in-pipe inspection. In Industrial Electronics, 1999. ISIE'99. Proceedings of the IEEE International Symposium on (Vol. 2, pp. 968-972). IEEE.

4. Nayak, A., \& Pradhan, S. K. (2014). Design of a new in-pipe inspection robot. Procedia Engineering, 97, 2081-2091.

5. Kwon, Y. S., Lim, H., Jung, E. J., \& Yi, B. J. (2008, May). Design and motion planning of a two-moduled indoor pipeline inspection robot. In Robotics and $\mathrm{Au}-$ tomation, 2008. ICRA 2008. IEEE International Conference on (pp. 3998-4004). IEEE.

6. Zhang, Y., Zhang, M., Sun, H., \& Jia, Q. (2010, October). Design and motion analysis of a flexible squirm pipe robot. In Intelligent System Design and Engineering Application (ISDEA), 2010 International Conference on (Vol. 1, pp. 527-531). IEEE.

7. Chablat, D., Venkateswaran, S., \& Boyer, F. (2018). Mechanical Design Optimization of a Piping Inspection Robot. Procedia CIRP, 70, pp. 307-312.

8. Chablat, D., Venkateswaran, S., \& Boyer, F. (2019). Dynamic Model of a BioInspired Robot for Piping Inspection. In ROMANSY 22 - Robot Design, Dynamics and Control (pp. 42-51). Springer, Cham.

9. Henry, R., Chablat, D., Porez, M., Boyer, F., \& Kanaan, D. (2014, August). Multiobjective design optimization of the leg mechanism for a piping inspection robot. In ASME 2014 International Design Engineering Technical Conferences and Computers and Information in Engineering Conference (pp. V05AT08A001-V05AT08A001).

10. Ryew, S., Baik, S. H., Ryu, S. W., Jung, K. M., Roh, S. G., \& Choi, H. R. (2000). Inpipe inspection robot system with active steering mechanism. In Intelligent Robots and Systems, 2000.(IROS 2000). Proceedings. 2000 IEEE/RSJ International Conference on (Vol. 3, pp. 1652-1657). IEEE.

11. Jha, R., Chablat, D., Baron, L., Rouillier, F., \& Moroz, G. (2018). Workspace, Joint space and Singularities of a family of Delta-Like Robot. Mechanism and Machine Theory, Vol.127, (pp. 73-95).

12. Moroz, G., Chablat, D., Wenger, P., \& Rouiller, F. (2010). Cusp points in the parameter space of RPR-2PRR parallel manipulators. In New Trends in Mechanism Science (pp. 29-37). Springer, Dordrecht.

13. Alici, G., \& Shirinzadeh, B. (2004). Topology optimisation and singularity analysis of a 3-SPS parallel manipulator with a passive constraining spherical joint. Mechanism and Machine Theory, 39(2), 215-235.

14. Bonev, I. A., Zlatanov, D., \& Gosselin, C. M. (2002, April). Advantages of the modified Euler angles in the design and control of PKMs. In 2002 Parallel Kinematic Machines International Conference (pp. 171-188).

15. van Riesen, A., Furet, M., Chevallereau, C., \& Wenger, P. (2019). Dynamic Analysis and Control of an Antagonistically Actuated Tensegrity Mechanism. In ROMANSY 22-Robot Design, Dynamics and Control (pp. 481-490). Springer, Cham.

16. Yigit, C. B., \& Boyraz, P. (2017). Design and Modelling of a Cable-Driven ParallelSeries Hybrid Variable Stiffness Joint Mechanism for Robotics. Mechanical Sciences, 8(1), 65-77. 